\title{
Tetraquarks in AdS/QCD
}

\author{
Hilmar Forkel* \\ Institut für Physik, Humboldt-Universität zu Berlin, D-12489 Berlin, Germany \\ E-mail: forkelaphysik.hu-berlin.de
}

\begin{abstract}
Multiquark correlations inside hadrons can have a significant and in some cases even striking impact on the hadron spectrum. We show how such correlations in general, and mesons with a dominant tetraquark content in particular, emerge holographically in the AdS/QCD framework. On this basis, we arrive at a holographic realization of an exceptionally strong four-quark binding and a correspondingly large tetraquark component in the lightest scalar mesons. Higher-lying tetraquark excitations, on the other hand, become too broad to form supernumeral scalar states.
\end{abstract}

Sixth International Conference on Quarks and Nuclear Physics

April 16-20, 2012

Ecole Polytechnique, Palaiseau, Paris

${ }^{*}$ Speaker. 


\section{Introduction}

A dominant tetraquark component is often invoked to explain non-standard features of mesons ranging from the lightest scalar nonet [1] to some of the recently discovered heavy-quark resonances [2]. Without guidance from the naive quark model, the theoretical analysis of such exotic states has to deal with the challenges of the strongly-coupled gauge dynamics more directly. Adding to the arsenal of suitable methods for this purpose (which now includes devoted lattice simulations [3]), the AdS/QCD approach [4] was recently extended to provide holographic insights into tetraquark properties [5].

The AdS/QCD program describes strong-interaction physics analytically by constructing approximate five-dimensional gravity duals for QCD on the basis of the gauge/string correspondence [6]. To access multiquark correlations inside hadrons within this framework, one exploits the fact that such correlations manifest themselves in the quark composition of the QCD interpolator which most strongly couples to the hadron under consideration. Hence these correlations leave a gaugeinvariant imprint on the interpolator's anomalous dimension which the AdS/CFT dictionary [6] translates into a multiquark-content-dependent mass correction for the hadron's dual bulk mode. This holographic mechanism was previously employed to analyze the impact of diquark correlations on masses and sizes of the light-quark baryons [7]. In the following, we will apply it to tetraquarks.

\section{Tetraquark holography}

The AdS/CFT dictionary associates the bulk mode $\varphi(x, z)$ (where $x$ are the coordinates along the boundary and $z$ denotes the orthogonal direction) dual to a given hadron $|h\rangle$ with the gaugetheory interpolator $J(x)$ which most strongly couples to $|h\rangle$. More specifically, for a scalar meson the scaling dimension $\Delta$ of $J$ determines the boundary condition [6]

$$
\varphi(x, z) \stackrel{z \rightarrow 0}{\longrightarrow} \varphi^{(0)}(x) z^{4-\Delta}
$$

for the dual bulk-mode solutions. This condition is imposed on the (at small $z$ leading) solutions of the bulk field equation by adjusting its mass term to

$$
m_{5}^{2} R^{2}=\Delta(\Delta-4)
$$

(where $m_{5}$ is the mode's mass and $R$ is the $\mathrm{AdS}_{5}$ curvature scalar). Interpolators with a larger quark content and correspondingly larger $\Delta$ are therefore related to heavier bulk excitations and generally to mesons with larger mass. (Note that the relation (2.2) is independent of the specific gravity dual under consideration).

In contrast to the unique quark-antiquark interpolator $J_{\bar{q} q}^{A}=\bar{q}^{a} t^{A} q^{a}$ for ordinary scalar mesons with $\Delta_{\bar{q} q}=3$, there are several independent (gauge-invariant and local) scalar four-quark operators with a common classical scaling dimension $\Delta_{\bar{q}^{2} q^{2}}=6$. (An explicit example is $J_{\bar{q}^{2} q^{2}}^{A}=$ $\varepsilon^{a b c} \varepsilon^{a d e} \bar{q}^{b} C \Gamma^{A} \bar{q}^{c} q^{d} C \Gamma^{A} q^{e}$ which contains a "good" (i.e. maximally attractive) diquark and a good antidiquark.) Since all of these four-quark operators couple to the scalar tetraquark state $|t\rangle$, we define the ("optimal") tetraquark interpolator $J_{\bar{q}^{2} q^{2}}$ as the particular linear combination which has maximal overlap $\left\langle 0\left|J_{\bar{q}^{2} q^{2}}\right| t\right\rangle$ with the tetraquark ground state. 
This definition encodes part of the tetraquark's structure into the field composition and anomalous dimension of $J_{\bar{q}^{2} q^{2}}$ since both are affected by the dominant quark couplings in $|t\rangle$. The anomalous dimension $\gamma(\mu)$, in particular, carries information on the multiquark correlations responsible e.g. for tetraquark binding [5]. Owing to the boundary condition (2.1) this information gets translated into a bulk mass correction $\Delta m_{5}(z)$ for the tetraquark's dual mode ${ }^{1}$. (The $z$ dependence of $\Delta m_{5}$ is inherited from the dependence of $\gamma$ on the renormalization scale $\mu \sim 1 / z$.)

Despite their rather straightforward origin, the implementation of these bulk-mass corrections into bottom-up duals is not without challenges. To begin with, at present QCD information on anomalous dimensions of hadronic interpolators is still scarce (especially in the infrared) and their renormalization scheme dependence raises compatibility issues. A more fundamental concern [5] is the naive AdS/QCD extrapolation of the multiquark physics with its pronounced $N_{c}$ dependence to $N_{c}=3$. Major benefits of the outlined mechanism, on the other hand, include that it will work in any of the current AdS/QCD duals, both non-dynamical (i.e. not backreacted) and dynamical (see e.g. Ref. [9]), and that the results are essentially model independent. In the following we will implement this mechanism into the "dilaton soft-wall" dual of Ref. [10] and apply it to the analysis of tetraquark correlations inside the lightest scalar mesons.

\section{Light scalar tetraquarks in the dilaton soft-wall dual}

The lightest scalar meson nonet (for reviews see e.g. [11]) has long been suggested to contain a dominant tetraquark component [1]. This at present arguably favored interpretation requires an exceptionally strong four-quark binding which should be large enough to reduce the tetraquark mass considerably below that of the lightest scalar $\bar{q} q$ mesons [12]. Hence light scalar tetraquarks furnish a particularly challenging testing ground for the holographic mechanism discussed above.

To implement the latter into an explicit dynamical framework, we adopt the soft-wall dual of Ref. [10] which contains the $\mathrm{AdS}_{5}$ metric and a quadratic dilaton field ${ }^{2} \Phi(z)=\lambda^{2} z^{2}$. After Fourier transforming the $x$ dependence of the tetraquark's dual modes $\varphi$ and defining a "reduced" scalar field $\phi(q, z)=(R / z)^{3 / 2} e^{-\lambda^{2} z^{2} / 2} \varphi(q, z)$, the radial bulk equation in this background turns into the Sturm-Liouville problem

$$
\left[-\partial_{z}^{2}+V(z)\right] \phi(q, z)=q^{2} \phi(q, z)
$$

with the potential

$$
V(z)=\left(\frac{15}{4}+m_{5}^{2} R^{2}\right) \frac{1}{z^{2}}+\lambda^{2}\left(\lambda^{2} z^{2}+2\right)
$$

For constant $m_{5}$ the normalizable solutions of this eigenvalue problem can be found analytically [5]. The resulting, discrete square-masses of the $n$-th radial excitations (related to $\Delta$ by Eq. (2.2)),

$$
M_{n}^{2}=q_{n}^{2}=4 \lambda^{2}\left(n+\frac{\Delta}{2}\right)
$$

lie on linear trajectories and indeed grow with $\Delta$.

In addition, the spectra (3.3) reveal that the ground state of the four-quark trajectory with $\Delta_{\bar{q}^{2} q^{2}}=6$ has a twice larger square mass $M_{\Delta=6,0}^{2}=2 M_{q \bar{q}, 0}^{2}$ than its $\Delta_{\bar{q} q}=3$ counterpart (as long

\footnotetext{
${ }^{1}$ For anomalous-dimension induced bulk-mass corrections in a different context see Ref. [8].

2 in contrast to the "metric soft wall" of Ref. [13]
} 
as anomalous dimensions are ignored). Simply replacing the conventional quark-antiquark interpolator by a $\bar{q}^{2} q^{2}$ interpolator would therefore result in four-quark states which are heavier, not lighter than the $\bar{q} q$ ground state. Of course, in view of our above discussion it is not surprising that the attraction required to bind tetraquarks is lacking. Indeed, if multiquark correlations generate the exceptional lightness of the tetraquark ground state, the latter should emerge from the so far neglected anomalous dimension of the tetraquark interpolator.

Inclusion of this anomalous dimension, which we denote by $\gamma(z)$, generalizes the bulk mass term (2.2) to

$$
m_{5}^{2}(z) R^{2}=[6+\gamma(z)][2+\gamma(z)]
$$

for the modes dual to the tetraquark interpolator $J_{\bar{q}^{2} q^{2}}$ with $\Delta_{\bar{q}^{2} q^{2}}=6+\gamma(z)$. This adds the universal correction

$$
\Delta V(z)=\gamma(z)[\gamma(z)+8] \frac{1}{z^{2}}
$$

to the potential (3.2) with $m_{5}^{2} R^{2}=12$. Note that this correction will be completely determined as soon as QCD information on the RG flow of $\gamma$ will become available. In the meantime, we resort to estimating its quantitative impact on the tetraquark spectrum and to deriving an exact lower bound on the ground-state mass. For the former purpose, we adopt the ansatz $\gamma(z)=-a z^{\eta}+b z^{\kappa}$ whose coefficients turn out to be tightly constrained by consistency and stability requirements [5].

A fundamental property of Eq. (3.5) is the lower bound $\Delta V(z) \geq-16 / z^{2}$ which holds for any $\gamma$ and prevents the collapse of dual modes into the $\mathrm{AdS}_{5}$ boundary. This bound is saturated by $\gamma \equiv-4$ and therefore yields the lower bound

$$
M_{\bar{q}^{2} q^{2}, 0} \geq M_{\Delta=2,0}=2 \lambda
$$

which determines the lightest tetraquark mass which Eq. (3.4) can generate. Moreover, for constant values of $\gamma$ in the range $-4<\gamma<-3$ the tetraquark's ground-state mass remains below its $\bar{q} q$ counterpart. Reassuringly, the underlying binding mechanism will work in other AdS/QCD duals as well. Indeed, $\gamma$ enters the bulk dynamics exclusively through the mass term (3.4) which is modelindependently prescribed by the AdS/CFT dictionary and yields the universal correction (3.5) in any AdS/QCD dual.

Higher-lying tetraquark excitations with masses $M_{\bar{q}^{2} q^{2}, n}$ (for $n>0$ ) beyond those of the corresponding quark-antiquark mesons require in addition a suitably running $\gamma(z)$. Our above ansatz, for example, can push the tetraquark masses $M_{\bar{q}^{2} q^{2}, n}$ beyond the $M_{\bar{q} q, n}$ from around $n \gtrsim 2$ while simultaneously generating an almost maximal ground-state binding energy. The latter drives $M_{\bar{q}^{2} q^{2}, 0}$ from $\sim 40 \%$ above (for $\gamma \equiv 0$ ) down to $\sim 20 \%$ below the $\bar{q} q$ ground-state $\operatorname{mass}^{4} M_{q \bar{q}, 0}=\sqrt{6} \lambda$. This can be seen in Fig. 1 where the corresponding spectra and potentials are plotted. It further appears that the higher-lying radial tetraquark excitations will be broad enough to prevent the appearance of supernumeral scalar states in the meson spectrum.

\footnotetext{
${ }^{3}$ Anomalous dimensions of a qualitatively similar $z$ dependence (in the region of interest) are encountered in dual backgrounds of holographic RG-flow type [14].

${ }^{4}$ Hence the phenomenological ratio $M_{\bar{q}^{2} q^{2}, 0} / M_{\bar{q} q, 0} \sim 0.8 / 1.5$ is not quantitatively reproduced, perhaps due to the neglect of the $\bar{q} q$ interpolator's anomalous dimension.
} 

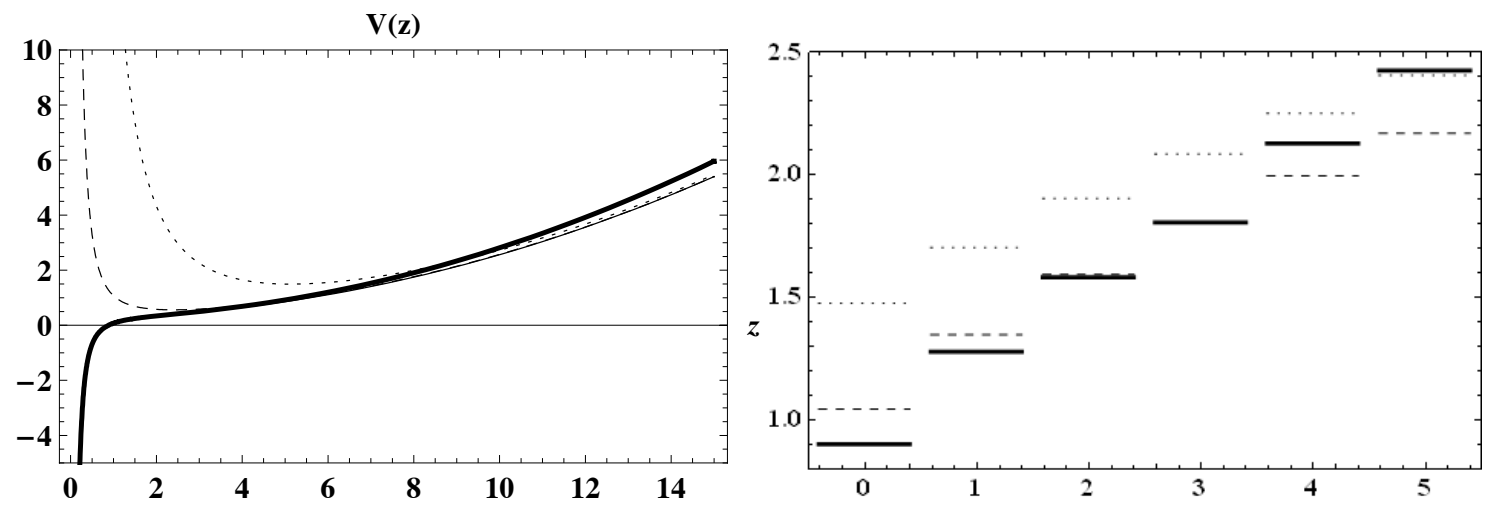

Figure 1: Left panel: the tetraquark's dual-mode potential (3.2) (i) for the anomalous dimension $\gamma(z)=$ $-a z^{\eta}+b z^{\kappa}$ with $a=4, b=0.05, \eta=0.001$ and $\kappa=2$ (thick line), (ii) for the four-quark interpolator with $\Delta=6, \gamma=0$ (dotted line), (iii) for the $\bar{q} q$ interpolator with $\Delta=3$ (dashed line) and (iv) for $\Delta=2$ (thin line) which saturates the Breitenlohner-Freedman bound. Right panel: The ground state $(n=0)$ and the first five excitations of the tetraquark mass spectrum (i) in the potential (i) of Fig. 1 (thick bars), (ii) for the $\bar{q} q$ interpolator (dashed bars) and (iii) for the $\Delta=6$ interpolator (dotted bars) with $\gamma=0$.

\section{Summary and conclusions}

We have presented an essentially model-independent extension of the AdS/QCD framework which describes multiquark correlations in hadrons by means of anomalous-dimension-induced bulk-mass corrections. This mechanism yields a robust holographic description of a dominant tetraquark component in the lowest-lying scalar mesons. Moreover, it provides an explicit lower bound on holographically attainable tetraquark masses. In the dilaton soft-wall gravity dual this bound sits at twice the infrared scale and can be almost saturated. The resulting, exceptionally strong four-quark binding renders the tetraquark ground-state nonet about $20 \%$ lighter than the lowest-lying scalar quark-antiquark state. Higher-lying tetraquark excitations, on the other hand, can be pushed beyond their quark-antiquark counterparts and are thus likely to dissolve into the multiparticle continuum.

\section{Acknowledgments}

It is a pleasure to thank the organizers of QNP 2012 for a very informative and enjoyable conference. Financial support from the Deutsche Forschungsgemeinschaft (DFG) is also acknowledged.

\section{References}

[1] R.L. Jaffe, Multiquark hadrons - Phenomenology of $Q^{2} \bar{Q}^{2}$ mesons, Phys. Rev. D 15, 267, 281 (1977); J.R. Peláez, Nature of light scalar mesons from their large- $N_{c}$ behavior, Phys. Rev. Lett. 92 (2004) 102001; G 't Hooft, G. Isidori, L. Maiani, A.D. Polosa and V. Riquer, A theory of scalar mesons, Phys. Lett. B 662 (2008) 424. 
[2] N. Brambilla et al., Heavy quarkonium: progress, puzzles, and opportunities, Eur. Phys. J. C 71, 1534 (2011); S. Godfrey and S. L. Olsen, The exotic XYZ charmonium-like mesons, Ann. Rev. Nucl. Part. Sci. 58 (2008) 51.

[3] S. Prelovsek et al., Lattice study of light scalar tetraquarks with $I=0,2,1 / 2,3 / 2$ : are $\sigma$ and $\kappa$ tetraquarks?, Phys. Rev. D 82 (2010) 094507; T. Kunihiro et al., The low-lying scalar mesons and related topics, Nucl. Phys. B (Proc. Suppl.) 186 (2009) 294; M. Loan, Z.-H. Luo and Y. Y. Lam, Lowest-lying tetraquark hadrons in anisotropic lattice QCD, Eur. Phys. J. C 57 (2008) 479; N. Mathur et al., Lattice QCD study of the scalar mesons $a_{0}(1450)$ and $\sigma(600)$, Phys. Rev. D 76 (2007) 114505.

[4] Y. Kim and D. Yi, Holography at work for nuclear and hadron physics, Adv. High Energy Phys. 2011 (2011) 259025; S.S. Gubser and A. Karch, From gauge-string duality to strong interactions: a Pedestrian's Guide, Annu. Rev. Nucl. Part. Sci. 59 (2009) 145; S.J. Brodsky and G.F. de Téramond, AdS/CFT and Light-Front QCD, arXiv:0802.0514; J. Erdmenger, N. Evans, I. Kirsch and E. Threlfall, Mesons in gauge/gravity duals - a review, Eur. Phys. J. A 35 (2008) 81.

[5] H. Forkel, Light scalar tetraquarks from a holographic perspective, Phys. Lett. B 694 (2010) 252; Multiquark correlations in light mesons and baryons from holographic QCD, AIP Conf. Proc. 1388 (2011) 182 [arXiv:1103.3902].

[6] O. Aharony et al., Large-N field theories, string theory and gravity, Phys. Rep. 323 (2000) 183.

[7] H. Forkel and E. Klempt, Diquark correlations in baryon spectroscopy and holographic QCD, Phys. Lett. B 679 (2009) 77.

[8] A. Vega and I. Schmidt, Modes with variable mass as an alternative in AdS/QCD models with chiral symmetry breaking, Phys. Rev. D 82 (2010) 115023.

[9] W. de Paula, T. Frederico, H. Forkel and M. Beyer, Dynamical AdS/QCD with area-law confinement and linear Regge trajectories, Phys. Rev. D 79 (2009) 075019; Solution of the 5D Einstein equations in a dilaton background model, Proc. Sci. (LC2008) (2008) 046 [arXiv: 0810 . 2710].

[10] A. Karch, E. Katz, D.T. Son and M.A. Stephanov, Linear Confinement and AdS/QCD, Phys. Rev. D 74 (2006) 015005.

[11] E. Klempt and A. Zaitsev, Glueballs, hybrids, multiquarks - Experimental facts versus QCD inspired concepts, Phys. Rep. 454, 1 (2007); C. Amsler and N.A. Törnqvist, Mesons beyond the naive quark model, Phys. Rep. 389, 61 (2004); D.V. Bugg, Four sorts of meson, Phys. Rept. 397, 257 (2004); F.E. Close and N.A. Törnqvist, Scalar mesons above and below $1 \mathrm{GeV}$, J. Phys. G 28, R249 (2002); T.V. Brito, F.S. Navarra, M. Nielsen and M.E. Bracco, QCD sum rule approach for the light scalar mesons as four-quark states, Phys. Lett. B 608 (2005) 69; E. Ruiz Arriola and W. Broniowski, Scalar-isoscalar states in the large- $N_{c}$ Regge approach, Phys. Rev. D. 81 (2010) 054009.

[12] A. Vega and I. Schmidt, Scalar hadrons in $A d S_{5} \times S^{5}$, Phys. Rev. D 78 (2008) 017703; P. Colangelo et al., Light scalar mesons in the soft-wall model of AdS/QCD, Phys. Rev. D 78 (2008) 055009; W. de Paula and T. Frederico, Scalar mesons within a dynamical holographic QCD model, Phys. Lett. B 693 (2010) 287.

[13] H. Forkel, M. Beyer and T. Frederico, Linear square-mass trajectories of radially and orbitally excited hadrons in holographic QCD, JHEP 07 (2007) 077; Linear meson and baryon trajectories in AdS/QCD, Intl. J.Mod. Phys. E 16 (2007) 2794; H. Forkel, Hadrons as Holograms, Phys. Rev. D 78 (2008) 025001; Proc. Sci., QCD-TNT09 (2009) 014 [arXiv: 0910.5955$].$

[14] W. Mück, Running scaling dimensions in holographic renormalization group flows, JHEP 08, 085 (2010). 